\section{A) Check for updates}

Cite this: Org. Biomol. Chem., 2020, 18,250

\title{
Photo-induced thiol-ene reactions for late-stage functionalization of unsaturated polyether macrocycles: regio and diastereoselective access to macrocyclic dithiol derivatives $\dagger+$
}

\author{
Elodie Brun, (DD ${ }^{a}$ Ke-Feng Zhang, ${ }^{a}$ Laure Guénée $^{\mathrm{b}}$ and Jérôme Lacour (D) *a
}

Received 3rd November 2019, Accepted 27th November 2019

DOI: $10.1039 / c 9 o b 02375$ e

rsc.li/obc

Double hydrothiolation of bis enol ether macrocycles was achieved under photo-mediated conditions. The thiol-ene reactions afford a fully regioselective anti-Markovnikov post-functionalization. Thanks to the use of ethanedithiol as reagent, moderate to excellent diastereoselectivity was accomplished leading to macrocycles containing four defined stereocenters in only three steps from 1,4-dioxane, tetrahydrofuran (THF) or tetrahydropyran (THP).

The thiol-ene reaction, which is the formal coupling of olefins and thiols to form alkylsulfide derivatives, is widely used in polymer science, nanoengineering, chemical biology and medicinal chemistry. ${ }^{1}$ This process, also known as hydrothiolation, is considered to be a click reaction and much of its success is related to this reactivity. ${ }^{2}$ With terminal olefins, the hydrothiolation can lead to two types of products namely, branched and linear sulfides with respect to position of the carbon-sulfur (C-S) bond formation. For most applications, the antiMarkovnikov thiol-ene reactivity, that generates only the latter class of linear products, is most interesting and sought for. This regioselectivity is usually achieved following free radical or base-induced mechanisms, and this under metal-free, metal-catalyzed, and photo-induced reaction conditions. ${ }^{1 a}$ However, stereochemical control of newly generated stereocenter(s) is often problematic and low to excellent selectivity can be obtained for a same substrate depending on the thiol reagent, reaction conditions and additives. ${ }^{3}$ This situation is even more complicated when multiple thiol-ene reactions can occur on a substrate carrying several olefins. ${ }^{4}$ In this context, our group recently reported the stereoselective deconjugations of bis- $\alpha, \beta$-unsaturated macrocycles $\mathbf{1}$ in presence of aromatic

\footnotetext{
${ }^{a}$ Department of Organic Chemistry, University of Geneva, Quai Ernest Ansermet 30, 1211 Geneva 4, Switzerland. E-mail: jerome.lacour@unige.ch

${ }^{b}$ Laboratory of Crystallography, University of Geneva, Quai Ernest Ansermet 24, 1211 Geneva 4, Switzerland

$\dagger$ In addition, the dataset for this article can be found at the following DOI: 10.26037/yareta:omb24lj5dbat3c6sqqtsecu4lu. It will be preserved for 10 years $\$$ Electronic supplementary information (ESI) available: Experimental conditions, ${ }^{1} \mathrm{H}$ NMR, ${ }^{13} \mathrm{C}$ NMR, ${ }^{19} \mathrm{~F}$ NMR and IR spectra of all new compounds; $R_{\mathrm{f}}$ and HR-MS. CCDC 1952403-1952406. For ESI and crystallographic data in CIF or other electronic format see DOI: 10.1039/c9ob02375e
}

amines and $t$-BuOK. ${ }^{5}$ In a single step (Scheme 1 , top), bis enol ether macrocycles are formed by double (tandem) amidations and olefin transpositions that yield products 2 as single stereoisomers (racemic, diastereomeric ratio $d r>49: 1$ ). Such bis (amide) derivatives 2 have been applied in fields ranging from chiroptical spectroscopy to molecular switches, nanosensors and heteroditopic receptors. ${ }^{6}$ These applications were achieved by the functionalization of the arylamide groups and not of the macrocyclic skeleton.

Herein, in an effort to achieve the late-stage functionalization of the unsaturated polyether fragments, double stereoselective hydrothiolations of compounds 2 are reported.

A

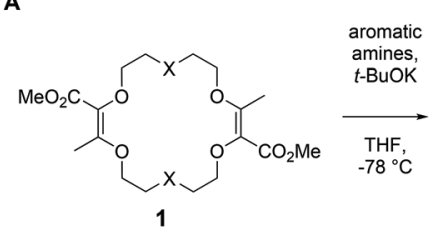

B

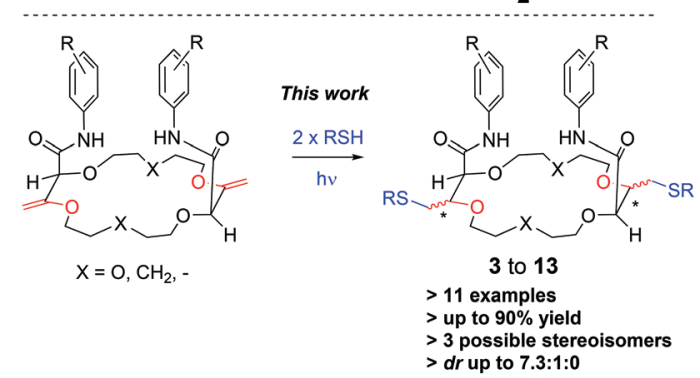

Scheme 1 Stereoselective synthesis of bis enol ether functionalized polyether macrocycles 2 (A, syn transposition, $d r>49: 1$ ). Regio and diastereoselective thiol-ene additions (B). 
Taking advantage of photo-initiated conditions, disulfides and dithiol derivatives $\mathbf{3}$ to $\mathbf{1 3}$ are generated in good to excellent yields and with moderate to excellent diastereocontrol ( $d r$ up to $7.3: 1: 0$, Scheme 1 and Fig. 2). The reaction is general and can be applied to a variety of macrocyclic precursors (16 and 18-membered rings, aromatic and heteroaromatic amides). To achieve higher selectivity, the use of bis thiol reagents was developed; the diastereoselectivity depending on the chain length between the two S-atoms. Overall, two acid-sensitive exocyclic enol ethers have been transformed selectively into saturated chemically-robust functional groups that can behave as handle for future developments. The thiol-ene reactions can be considered orthogonal as the components react together in high yields and in the presence of the other functional groups and chromophores. ${ }^{7}$

Previously, it was shown that methyl $\alpha$-diazo- $\beta$-ketoester reacts with THF, 1,4-dioxane or THP under dirhodium catalysis in formal $[3+Y+3+Y]$ multi-component condensations $(Y=5$ or 6). The process is mild and affords the unsaturated macrocycles 1 on multi-gram scale (up to 20 grams) while using a low catalyst loading (0.01-0.001 mol\%). ${ }^{8}$ As mentioned earlier, compounds 1 react with excesses of $\mathrm{ArNH}_{2}$ and $t$-BuOK ( $>3$ equiv. each) to yield unsaturated bis(amide) derivatives 2 . This reaction tolerates a large variety of aromatic amines. ${ }^{5}$ However, despite major efforts in the group, it was never possible to achieve the late stage functionalization of the exocyclic terminal olefins. Bis enol ethers 2 are particularly sensitive to Lewis and Brønsted acidic conditions (or workup). Care was thus taken to study the reactivity of compounds 2 under photo-induced thiol-ene conditions that are neutral essentially.

In a first attempt, the reaction was investigated in the presence of 2,2-dimethoxyphenylacetophenone (DMPA) as photoinitiator and under mercury lamp irradiation. ${ }^{9}$ Quite a few reactions were performed using macrocycle $2(\mathrm{X}=\mathrm{O})$ as model substrate, carrying two 3,5-bis(trifluoromethyl)phenyl carboxamide groups, and various thiols as reagents $(\mathbf{1 4 a - 1 4 h}$, Table 1). With thiolacetic acid 14a, the reaction worked smoothly giving only the corresponding anti-Markovnikov addition product $3 \mathrm{a}$ in $90 \%$ yield within 1 hour (entry 1). However, 3a was obtained as a mixture of three diastereoisomers as a consequence of the creation of two new stereogenic centers and the presence of a local symmetry. The three stereoisomers could be separated by preparative thin layer chromatography. By NMR spectroscopy, the two $C_{2}$-symmetric derivatives were readily differentiated from the single $C_{1}$ isomer. The assignment of their relative cis and trans configurations was achieved upon deprotection of the acetyl groups $\left(\mathrm{K}_{2} \mathrm{CO}_{3} 4.0\right.$ equiv., $\left.\mathrm{MeOH} / \mathrm{THF} / \mathrm{H}_{2} \mathrm{O}, 25{ }^{\circ} \mathrm{C}, 1 \mathrm{~h}\right)$. The resulting $C_{2}$-cis-4 and $C_{2}$-trans $-\mathbf{4}$ bis thiols were found to be crystalline and structures were unambiguously determined by X-ray crystallography (Fig. 1). While the $C_{2}$-symmetry of cis-4 was maintained in the solid state, a non-symmetric conformation was found for trans-4, ${ }^{10}$ unlike in solution for which duplicated functional groups are magnetically equivalent in the ${ }^{1} \mathrm{H}$ and ${ }^{13} \mathrm{C}$ NMR spectra. This signifies either fast positional inter-
Table 1 Optimization studies ${ }^{a}$
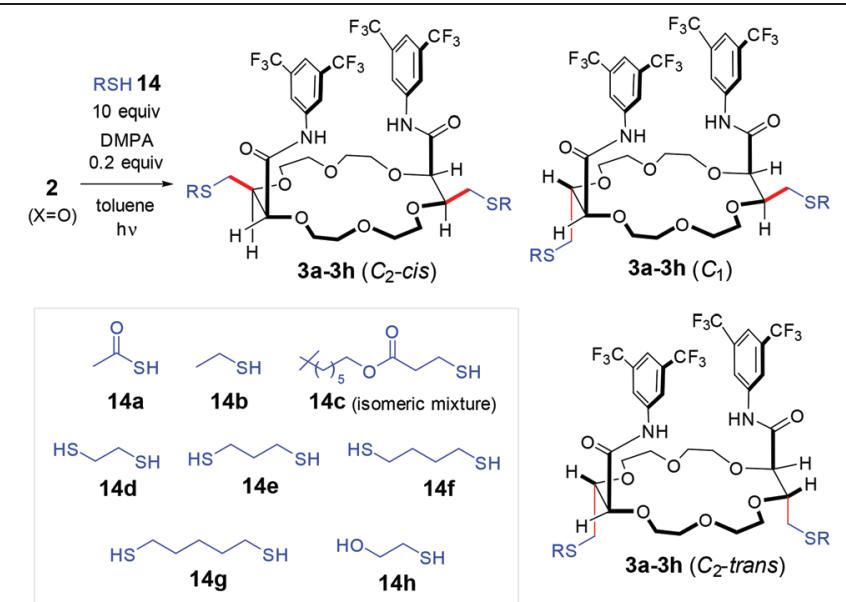

\begin{tabular}{lllllll}
\hline Entry & RSH, 14 & Time (h) & $d r^{b, c}$ & $C_{2}$-cis $^{d}$ & $C_{1}{ }^{d}$ & $C_{2}$-trans $^{d}$ \\
\hline 1 & $\mathbf{1 4 a}$ & 1 & $1.5: 1.1: 1^{c}$ & $38 \%$ & $27 \%$ & $25 \%$ \\
2 & $\mathbf{1 4 b}$ & 1 & $1.8: 1: 0^{c}$ & $48 \%$ & $26 \%$ & - \\
3 & $\mathbf{1 4 c}$ & 1.5 & $1.9: 1: 0^{c}$ & $49 \%$ & $26 \%$ & - \\
4 & $\mathbf{1 4 d}$ & 2 & $7.3: 1: 0^{b}$ & $57 \%$ & n.d. & - \\
5 & $\mathbf{1 4}$ & 2 & $4.0: 1: 0^{b}$ & $60 \%$ & n.d. & - \\
6 & $\mathbf{1 4 f}$ & 36 & $2.0: 1: 0^{b}$ & $27 \%$ & $15 \%$ & - \\
7 & $\mathbf{1 4 g}$ & 36 & $1.5: 1: 0^{b}$ & $34 \%$ & $21 \%$ & - \\
8 & $\mathbf{1 4 h}$ & 4 & $2.2: 2.4: 1^{c}$ & $29 \%$ & $31 \%$ & $13 \%$ \\
$9^{e}$ & $\mathbf{1 4 a}$ & 2 & $1: 1.2: 1.4^{c}$ & $22 \%$ & $26 \%$ & $31 \%$
\end{tabular}

${ }^{a}$ Reactions were performed with $0.1 \mathrm{mmol}$ of $2 .{ }^{b}$ Diastereomeric ratio $C_{2}$-cis : $C_{1}: C_{2}$-trans determined by ${ }^{1} \mathrm{H}$-NMR analysis of crude mixtures when possible. ${ }^{c} d r$ based on isolated fraction yields. ${ }^{d}$ Isolated yields. ${ }^{e}$ Reaction conditions: $2(0.1 \mathrm{mmol}), 14 \mathrm{a}$ (4.0 equiv.), $\mathrm{Et}_{3} \mathrm{~B}(1.0 \mathrm{M}$ in hexane, 3.2 equiv.), TBC (2.4 equiv.), $\mathrm{CH}_{2} \mathrm{Cl}_{2}(0.15 \mathrm{~mL})$, r.t., under air, 2.0 h. n.d. stands for not determined.

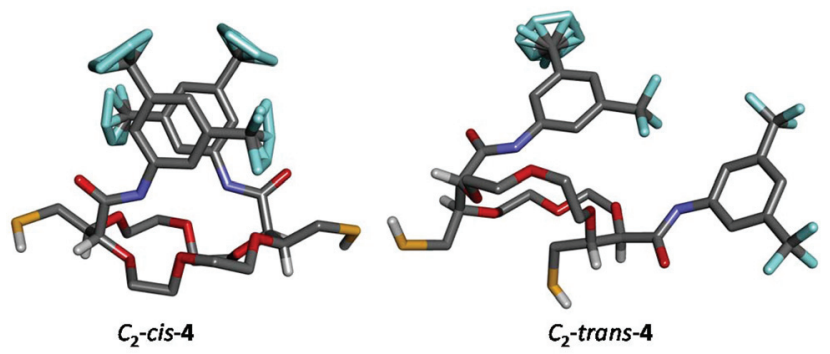

Fig. 1 Stick view of the crystal structures of $C_{2}$-cis-4 and $C_{2}$-trans-4. For clarity reasons, most hydrogen atoms are omitted. Disorder is observed for some $\mathrm{CF}_{3}$ groups.

changes on the NMR time scale or the adoption of a $C_{2}$-symmetric conformation in solution. ${ }^{11}$

Care was then taken to increase the diastereoselectivity. As expected, classical thiol reagents participated to the reaction and, to our delight, an improvement was afforded with ethanethiol 14b and methylheptyl 3-mercaptopropanoate 14c. In these cases, only two instead of three products were formed in ca. 1.8:1:0 ratio. The major isomer of $3 \mathbf{b}$ presents a $C_{2}$-cis configuration, as determined by the X-ray crystallography, and 
the minor one is the $C_{1}$ product. ${ }^{12}$ Looking to improve the selectivity further and in view of the expected radical mechanism (vide infra), we turned our attention to dithiol derivatives as reagents. ${ }^{4,13}$ In thiol-ene reactions, it was our analysis that one of the two sulfur atoms would participate in the $\mathrm{C}-\mathrm{S}$ bond forming reaction (propagation step) while the other could contribute as a $\mathrm{H}$-donor atom. It was thus imagined that an intramolecular chain transfer step could favor an enhanced stereoselectivity; the discrimination depending then probably on the chain length between the two S-atoms. Several dithiols were selected, namely 14d-14g. With shorter ethanedithiol 14d, an excellent diastereoselectivity was obtained $(7.3: 1: 0 d r$, entry 4$).{ }^{14}$ Remarkably, step-by-step increase of the chain length between the thiols resulted in lower and lower diastereoselectivity ratios (Table 1, entries 4-7). With 2-mercaptoethan-1-ol $\mathbf{1 4 h}$, poor selectivity was again obtained (2.2:2.4:1 $d r$, entry 8). ${ }^{15}$ All these results show the importance of the chain transfer step in the stereodetermining events. Finally, inspired by Renaud's studies on the $\mathrm{Et}_{3} \mathrm{~B}$-mediated radical chemistry ${ }^{16}$ we wondered whether these mild and efficient conditions could improve or modify the selectivity. With thiolacetic acid 14a, a mixture of three stereoisomers was again obtained in essentially an equimolar ratio (entry 9).

With the optimal conditions for the thiol-ene reaction in hand (ethanedithiol 14b, Table 1, entry 4), the generality and limitations of the process were tested. Macrocycles 2 derived from THP $\left(\mathrm{X}=\mathrm{CH}_{2}\right)$ and THF $(\mathrm{X}=-)$ were reacted to afford the corresponding 18C4 and 16C4 dithiols 5 and 6 (Fig. 2). Only two stereoisomers albeit with lower diastereoselectivity $(d r$ $4.0: 1$ and 2.6:1) and yields were obtained in favor of the major $C_{2}$-cis derivatives ( $41 \%$ and $32 \%$ respectively). This shows, not too surprisingly, that the diastereoselectivity is influenced by the nature and size (conformation) of the starting macrocycles 2. Next, various substrates with heteroaromatic substituents were tested. In such instances, longer reaction times (15-36 h) were required to achieve full conversions. Reactions proceeded well with bis para and meta pyridines affording products 7 and 8 in good yields (64-73\%) but moderate selectivity ( $d r c a$. 3.2:1:0). When bis pyrimidine substituents were used, 9 was obtained in 70\% yield and a 4.0:1:0 diastereomeric ratio. However, in the presence of ortho pyridines (product 10), three diastereomers were observed in $2.3: 1.3: 1$ ratio among the $C_{2}$-cis, $C_{1}$ and $C_{2}$-trans isomers. Currently, to explain this change in selectivity, only a proximity effect can be tentatively evoked with a participation of the Lewis basic pyridine nitrogen atoms that would interact with the pendant thiol groups.

Then, we examined substrates carrying polyarenes, namely 1-naphthyl, 2-fluorenyl and 1-pyrenyl amide substituents. Under standard reaction conditions, only two diastereomers and good yields were obtained in all cases. With naphthalene and pyrene derivatives, dithiols $\mathbf{1 1}$ and $\mathbf{1 2}$ afforded the $C_{2}$-cis isomer as the major component in a 1.6:1 ratio only; a slightly higher diastereoselectivity $(2.1: 1)$ was observed with $9 H$-fluorene 13. Finally, a macrocycle 2 bearing two perylene units was also tested as substrate. However, conversion could not be
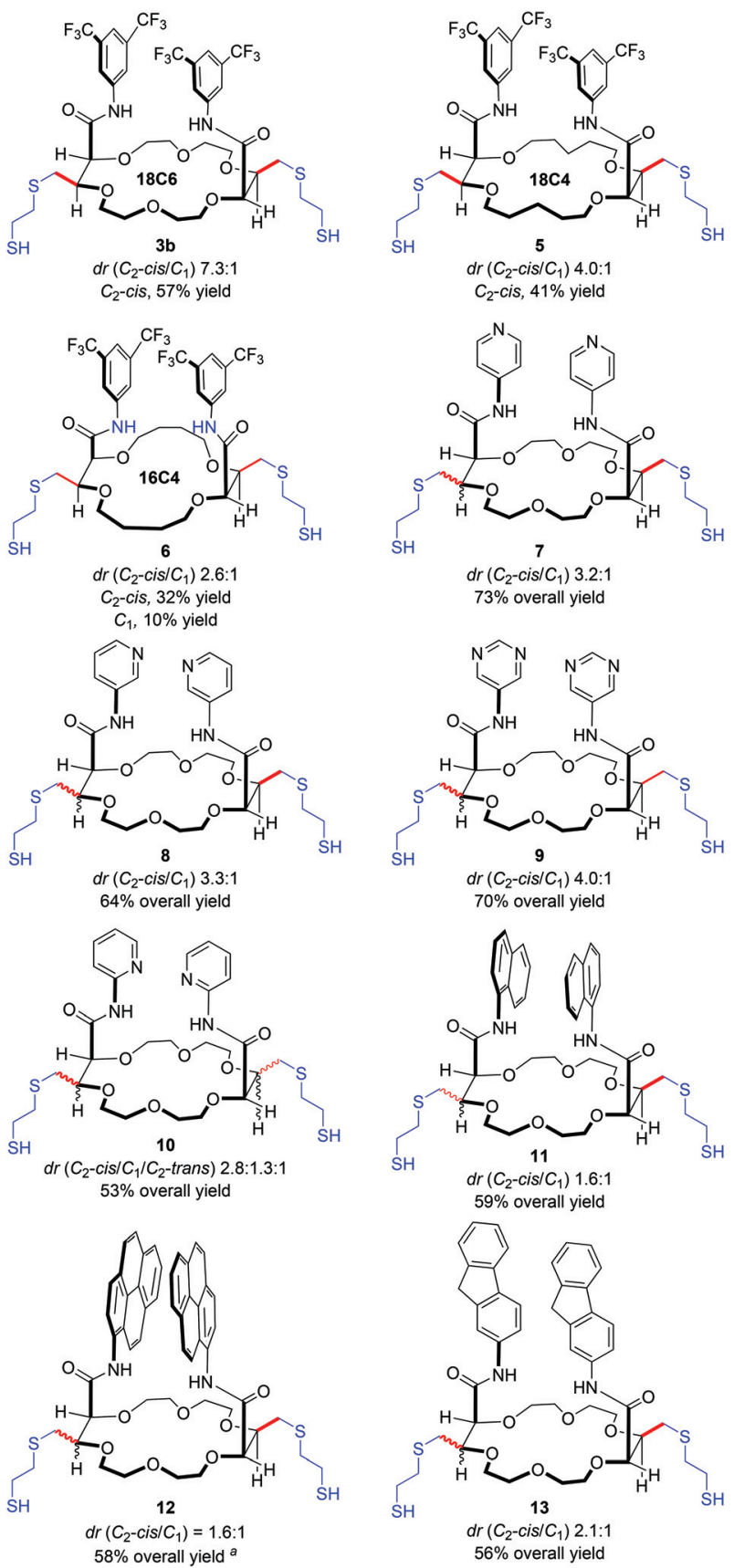

Fig. 2 Substrate scope. ${ }^{a}$ Reaction performed in degassed THF rather than toluene.

achieved using the current conditions; the perylene chromophores perturbing the photo-induced process.

Based on previous studies, a mechanistic rationale can be proposed for the thiol-ene reaction. ${ }^{1 a_{f} f}$ In Scheme 2, it is exemplified with ethanedithiol 14d that gives the higher level of stereocontrol. Upon photo-initiation with cleavable DMPA, thiyl radicals $\mathbf{A}$ are readily generated. These moieties add to the less substituted ends of the enol ether $\mathrm{C}=\mathrm{C}$ bonds, forming carbon-centered radicals $\mathbf{B}$ reversibly. ${ }^{1 f}$ Free radicals $\mathbf{B}$ then abstract intramolecularly hydrogen atoms from the 

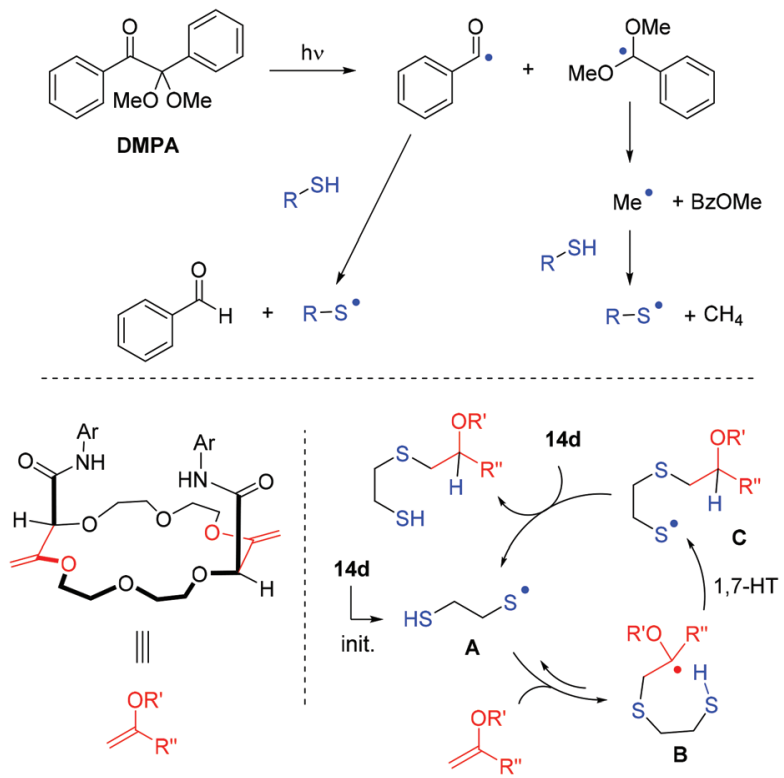

Scheme 2 Mechanistic proposal. HT stands for hydrogen transfer.

pendant thiol functional groups to deliver intermediates $\mathbf{C}$. Then, reactions with 14d afford the targeted sulfide products along with the regenerated thiyl radical $\mathbf{A}$ that propagates the chain reaction. In fact, due to the presence of the second thiol group in reagents $\mathbf{1 4 d - 1 4 g}$, intramolecular mechanisms are probably favored for the hydrogen atom abstraction. However, in such intramolecular steps, geometric factors are known to dominate. $^{17}$ In our system, the shortest possible intramolecular process is a 1,7-hydrogen atom transfer with 14d that leads to the highest selectivity. ${ }^{18}$ With an increasing chain length between the two S-atoms, entropies of activation for the intramolecular process become less favorable and this may be the reason for the lower selectivity ratios. ${ }^{19}$ Reversible hydrogen atom abstractions adjacent to ethereal oxygen atoms have been reported with thiols and can lead to the epimerization of stereogenic centers but such reactions occur under more strenuous conditions (octane, $125{ }^{\circ} \mathrm{C}$ ) than that employed. ${ }^{20}$ A thermodynamic control for step $\mathbf{B}$ to $\mathbf{C}$ and the issuing stereoselectivity are thus unlikely in the present situation.

In conclusion, double hydrothiolation of bis enol ether macrocycles 2 was achieved under photo-induced radicalbased conditions. The straightforward post-functionalization occurred with full anti-Markovnikov regioselectivity. Thanks to the use of ethanedithiol as reagent, $C_{2}$-trans isomers were essentially removed from the reaction mixtures (with one exception). This greatly simplifies the isolation and purification of the remaining $C_{2}$-cis and $C_{1}$ isomers by chromatography. Overall, moderate to excellent diastereoselectivity could be accomplished leading to macrocycles containing four defined stereogenic centers in only three steps from 1,4dioxane, THF or THP. The obtained products are currently considered for various applications in which the newly-introduced sulfur containing groups can bring reactivity and selectivity in intermolecular interactions.

\section{Conflicts of interest}

There are no conflicts to declare.

\section{Acknowledgements}

We thank the University of Geneva and the Swiss National Science Foundation for financial support (SNF 200020-172497 and 200020-184843). We acknowledge the contributions of the Sciences Mass Spectrometry (SMS) platform at the Faculty of Sciences, University of Geneva. We thank Professor Philippe Renaud (Bern), Valentin Soulard and Dr Qi Huang for their assistance in the $\mathrm{Et}_{3} \mathrm{~B}$-mediated chemistry. We thank them and Dr Fabrice Dénès (Nantes) for their mechanistic insight.

\section{Notes and references}

1 (a) A. K. Sinha and D. Equbal, Asian J. Org.Chem., 2019, 8, 32-47; (b) C. Ellingford, C. Bowen, T. McNally and C. Wan, Macromol. Rapid Commun., 2018, 39, 1800340; (c) R. Kowalczyk, P. W. R. Harris, G. M. Williams, S.-H. Yang and M. A. Brimble, in Peptides and Peptide-based Biomaterials and their Biomedical Applications, ed. A. Sunna, A. Care and P. L. Bergquist, Springer International Publishing, Cham, 2017, pp. 185-227; (d) M. Firdaus, Asian J. Org. Chem., 2017, 6, 1702-1714; (e) N. B. Cramer and C. N. Bowman, in Chemoselective and Bioorthogonal Ligation Reactions, ed. W. R. Algar, P. E. Dawson and I. L. Medintz, 2017, pp. 117-145; ( $f$ ) F. Dénès, M. Pichowicz, G. Povie and P. Renaud, Chem. Rev., 2014, 114, 2587-2693.

2 (a) A. B. Lowe, Polym. Chem., 2014, 5, 4820-4870; (b) C. E. Hoyle and C. N. Bowman, Angew. Chem., Int. Ed., 2010, 49, 1540-1573.

3 (a) M. Bege, I. Bereczki, M. Herczeg, M. Kicsák, D. Eszenyi, P. Herczegh and A. Borbás, Org. Biomol. Chem., 2017, 15, 9226-9233; (b) D. Limnios and C. G. Kokotos, Adv. Synth. Catal., 2017, 359, 323-328; (c) L. Lázár, M. Csávás, Á. Hadházi, M. Herczeg, M. Tóth, S. László, T. Barna, P. Herczegh and A. Borbás, Org. Biomol. Chem., 2013, 11, 5339-5350.

4 H. Tagoshi and T. Endo, Chem. Lett., 1987, 16, 2363-2364.

5 M. Vishe, R. Hrdina, A. I. Poblador-Bahamonde, C. Besnard, L. Guénée, T. Bürgi and J. Lacour, Chem. Sci., 2015, 6, 4923-4928.

6 (a) Z. Jarolimova, M. Vishe, J. Lacour and E. Bakker, Chem. Sci., 2016, 7, 525-533; (b) S. Sinn, F. Biedermann, M. Vishe, A. Aliprandi, C. Besnard, J. Lacour and L. De Cola, ChemPhysChem, 2016, 17, 1829-1834; (c) S. K. Ray, A. Homberg, M. Vishe, C. Besnard and J. Lacour, Chem. Eur. J., 2018, 24, 2944-2951; (d) M. Vishe, T. Lathion, S. Pascal, O. Yushchenko, A. Homberg, E. Brun, C. Piguet, E. Vauthey and J. Lacour, Helv. Chim. Acta, 2018, 101, e1700265; (e) A. Homberg, E. Brun, F. Zinna, S. Pascal, M. Górecki, L. Monnier, C. Besnard, G. Pescitelli, L. Di Bari and J. Lacour, Chem. Sci., 2018, 9, 7043-7052; (f) A. Aster, 
G. Licari, F. Zinna, E. Brun, T. Kumpulainen, E. Tajkhorshid, J. lacour and E. Vauthey, Chem. Sci., 2019, 10, 10629-10639; (g) A. Homberg, R. Hrdina, M. Vishe, L. Guénée and J. Lacour, Org. Biomol. Chem., 2019, 17, 6905-6910; (h) F. Zinna, S. Voci, L. Arrico, E. Brun, A. Homberg, L. Bouffier, T. Funaioli, J. Lacour, N. Sojic and L. Di Bari, Angew. Chem., Int. Ed., 2019, 58, 6952-6956.

7 C.-H. Wong and S. C. Zimmerman, Chem. Commun., 2013, 49, 1679-1695.

8 (a) A. Homberg, D. Poggiali, M. Vishe, C. Besnard, L. Guénée and J. Lacour, Org. Lett., 2019, 687-691; (b) D. Poggiali, A. Homberg, T. Lathion, C. Piguet and J. Lacour, ACS Catal., 2016, 6, 4877-4881; (c) M. Vishe, R. Hrdina, L. Guénée, C. Besnard and J. Lacour, Adv. Synth. Catal., 2013, 355, 3161-3169; (d) W. Zeghida, C. Besnard and J. Lacour, Angew. Chem., Int. Ed., 2010, 49, 7253-7256.

9 H. Fischer, R. Baer, R. Hany, I. Verhoolen and M. Walbiner, J. Chem. Soc., Perkin Trans. 2, 1990, 787-798.

10 A non-symmetric conformation was also found for $C_{2}$-trans3a. See the ESI and CCDC 1952405.

11 Of the two hypotheses, the second is judged more likely but one hypothesis does not preclude the other.

12 In comparison with unsaturated $2(\mathrm{X}=\mathrm{O})$ which assumes an overall planar geometry for the macrocyclic platform, cis-3b and cis-4 present curved $C_{2}$-symmetric basket-like structures for the heterocyclic macrocycle.

13 For the use of the monosodium salt of 1,2-ethanedithiol as reducing agent, see: Y. Ando, S. Hori, T. Fukazawa, K. Ohmori and K. Suzuki, Angew. Chem., Int. Ed., 2015, 54, 9650-9653.

14 The high selectivity might be the result of the double addition and of the Horeau principle: A. M. Harned,
Tetrahedron, 2018, 74, 3797-3841; V. Rautenstrauch, Bull. Soc. Chim. Fr., 1994, 131, 515-524; J. P. Vigneron, M. Dhaenens and A. Horeau, Tetrahedron, 1973, 29, 1055-1059. Unfortunately, in this instance, we cannot generate analogous substrates with a single enol ether functional group that would allow to test the selectivity for the mono-addition.

15 Dissociation energies of $\mathrm{O}-\mathrm{H}$ bonds $\left(104.4 \mathrm{kcal} \mathrm{mol}^{-1}\right.$, $\mathrm{MeOH})$ are quite higher than that of thiols $\left(88 \mathrm{kcal} \mathrm{mol}^{-1}\right.$, $\mathrm{MeSH}$ ). As such, and contrary to the later discussion in Scheme 2, an intermolecular mechanism is most likely in that case. Stereoselectivity differences between $\mathbf{1 4 h}$ and monothiols 14b-14c are then difficult to explain.

16 (a) G. Povie, S. R. Suravarapu, M. P. Bircher, M. M. Mojzes, S. Rieder and P. Renaud, Sci. Adv., 2018, 4, eaat6031; (b) G. Povie, A.-T. Tran, D. Bonnaffé, J. Habegger, Z. Hu, C. Le Narvor and P. Renaud, Angew. Chem., Int. Ed., 2014, 53, 3894-3898.

17 (a) D. Denenmark, P. Hoffmann, T. Winkler, A. Waldner and A. De Mesmaeker, Synlett, 1991, 621-624; (b) B. Viskolcz, G. Lendvay, T. Körtvélyesi and L. Seres, J. Am. Chem. Soc., 1996, 118, 3006-3009.

18 D. Denenmark, T. Winkler, A. Waldner and A. De Mesmaeker, Tetrahedron Lett., 1992, 33, 3613-3616.

19 (a) A. E. Dorigo and K. N. Houk, J. Am. Chem. Soc., 1987, 109, 2195-2197; (b) A. E. Dorigo, M. A. McCarrick, R. J. Loncharich and K. Houk, J. Am. Chem. Soc., 1990, 112, 7508-7514.

20 (a) H.-S. Dang, B. P. Roberts and D. A. Tocher, J. Chem. Soc., Perkin Trans. 1, 2001, 2452-2461; (b) H.-S. Dang and B. P. Roberts, Tetrahedron Lett., 2000, 41, 8595-8599; (c) H.-S. Dang and B. P. Roberts, Tetrahedron Lett., 1999, 40, 4271-4274. 